\title{
Birthweight and early-onset type 2 diabetes in American Indians: differential effects in adolescents and young adults and additive effects of genotype, BMI and maternal diabetes
}

\author{
Muideen T. Olaiya $^{1} \cdot$ Lauren E. Wedekind $^{1,2} \cdot$ Robert L. Hanson $^{1} \cdot$ Madhumita Sinha $^{1} \cdot$ Sayuko Kobes $^{1}$. \\ Robert G. Nelson ${ }^{1} \cdot$ Leslie J. Baier $^{1}$ • William C. Knowler ${ }^{1}$
}

Received: 13 February 2019 / Accepted: 23 April 2019 / Published online: 20 May 2019

(C) This is a U.S. government work and not under copyright protection in the U.S.; foreign copyright protection may apply 2019

\begin{abstract}
Aims/hypothesis The aim of this work was to estimate the impact of birthweight on early-onset (age $<40$ years) type 2 diabetes. Methods A longitudinal study of American Indians, aged $\geq 5$ years, was conducted from 1965 to 2007 . Participants who had a recorded birthweight were followed until they developed diabetes or their last examination before the age of 40 years, whichever came first. Age- and sex-adjusted diabetes incidence rates were computed and Poisson regression was used to model the effect of birthweight on diabetes incidence, adjusted for sex, BMI, a type 2 diabetes susceptibility genetic risk score (GRS) and maternal covariates.

Results Among 3039 participants, there were 652 incident diabetes cases over a median follow-up of 14.3 years. Diabetes incidence increased with age and was greater in the lowest and highest quintiles of birthweight. Adjusted for covariates, the effect of birthweight on diabetes varied over time, with a non-linear effect at 10-19 years $(p<0.001)$ and a negative linear effect at older age intervals (20-29 years, $p<0.001 ; 30-39$ years, $p=0.003)$. Higher GRS, greater BMI and maternal diabetes had additive but not interactive effects on the association between birthweight and diabetes incidence.

Conclusions/interpretation In this high-risk population, both low and high birthweights were associated with increased type 2 diabetes risk in adolescence (age 10-19 years) but only low birthweight was associated with increased risk in young adulthood (20-39 years). Higher type 2 diabetes GRS, greater BMI and maternal diabetes added to the risk of early-onset diabetes.
\end{abstract}

Keywords Birthweight · Diabetes mellitus · Genetic susceptibility · Incidence study · Type 2

\section{Abbreviations \\ GRS Genetic risk score \\ IRR Incidence rate ratio}

Electronic supplementary material The online version of this article (https://doi.org/10.1007/s00125-019-4899-9) contains peer-reviewed but unedited supplementary material, which is available to authorised users.

Muideen T. Olaiya

muideen.olaiya@nih.gov

1 Phoenix Epidemiology and Clinical Research Branch, National Institute of Diabetes and Digestive Kidney Diseases, National Institutes of Health, 1550 East Indian School Road,

Phoenix, AZ 85014, USA

2 Nuffield Department of Medicine, University of Oxford, Oxford, UK

\section{Introduction}

Associations between birthweight and later type 2 diabetes mellitus are well documented [1-4]. In 1991, Hales et al observed an inverse association between birthweight and the prevalence of type 2 diabetes among men aged 64 years in the UK [1]. By contrast, in 1994, we reported a non-linear association between birthweight and the prevalence of type 2 diabetes in Pima Indians aged 20-39 years, with greater type 2 diabetes prevalence observed in people with low or high birthweight $(\mathrm{U}$ shaped association) [2]. These earlier findings, and those of many subsequent studies [3, 4], however, were based largely on prevalence data obtained from adults ( $\geq 40$ years in many cases) and thus may not help to explain the currently increasing incidence of type 2 diabetes in the younger population [5, 6].

The association between birthweight and later type 2 diabetes has been attributed to environmental factors [7] and 


\section{Research in context}

\section{What is already known about this subject?}

- $\quad$ Evidence on the association between birthweight and type 2 diabetes is limited by inconsistent findings and lack of prospective data on diabetes incidence; therefore, little is known about the impact of birthweight on early-onset (age $<40$ years) type 2 diabetes

- Most of the genetic variations underlying the association between birthweight and type 2 diabetes are not well understood as the few known variants account for $<5 \%$ of heritability for type 2 diabetes

\section{What is the key question?}

- What is the effect of birthweight on the development of early-onset type 2 diabetes and does the aggregate contribution of established type 2 diabetes susceptibility variants modify this effect?

\section{What are the new findings?}

- Prospective analyses revealed differential effects of birthweight on type 2 diabetes risk: a non-linear (U shaped) effect in adolescents (10-19 years) but a negative linear effect in young adults (20-39 years)

- High type 2 diabetes genetic risk score, BMI and in utero exposure to diabetes have additive rather than interactive effects on birthweight-associated early-onset type 2 diabetes risk

How might this impact on clinical practice in the foreseeable future?

- The effect of high birthweight on type 2 diabetes risk, which occurs largely at young ages, may be driven by an intergenerational vicious cycle of type 2 diabetes and obesity

- Children with a high birthweight who were born to mothers with diabetes are at especially high risk of type 2 diabetes and should be closely monitored for obesity and diabetes

genetic factors $[2,8-11]$. Our previous report of the association between paternal diabetes and low birthweight provided early evidence on the potential influence of genetic factors on the association between birthweight and later type 2 diabetes [10]. In support of this observation, some type 2 diabetes susceptibility genetic variants were found to be associated with either low birthweight (e.g. ADCY5, CDKAL1 and $H H E X-I D E)[12,13]$ or high birthweight (e.g. ANK1, $M T N R 1 B$ and $A B C C 8)[12,14]$. However, these genetic variants have thus far accounted for $<5 \%$ of heritability for type 2 diabetes [13].

Pima Indians living in south-western USA have a high prevalence of type 2 diabetes $[15,16]$, and an increasing incidence of type 2 diabetes in youth (aged $<25$ years) $[17,18]$. In this population, we estimated the effect of birthweight on the incidence of early-onset type 2 diabetes (aged $<40$ years). We also determined the extent to which the aggregate contribution of established type 2 diabetes susceptibility genetic variants modifies the effect of birthweight on the risk of early-onset type 2 diabetes.

\section{Methods}

Study design and participants Residents of the Gila River Indian Community in Arizona, aged $\geq 5$ years, participated in a longitudinal study of diabetes and related complications from 1965 to 2007. Diabetes in this community is thought to be exclusively type 2 diabetes, with no evidence of the autoimmunity typical of type 1 diabetes, even in those with very early onset [19]. Participants were asked to undertake standardised health examinations biennially. The present analysis comprised participants who met the following criteria: (1) were singleton births and had a record of birthweight; (2) reported at least 50\% heritage from the Pima or closely related Tohono O'odham Indian tribe; (3) had at least two research examinations, each of which included an OGTT and measurements of height and weight and (4) had genome-wide genotypic data and complete data on other covariates excluding paternal variables (see the description of covariates below). This study was approved by the Institutional Review Board of the National Institute of Diabetes and Digestive and Kidney Diseases. Participants aged $<18$ years gave their assent to participate in the study, in addition to written informed consent obtained from parents. Participants aged $\geq 18$ years provided written informed consent.

Data collection Data on birthweight were obtained from birth certificates and review of medical records. Anthropometric and biochemical data on participants and their parents were obtained from their respective research examinations. BMI was calculated from height and weight measured while 
participants were dressed in light clothing and without shoes. A modified OGTT was conducted by measuring the plasma glucose at $2 \mathrm{~h}$ following a $75 \mathrm{~g}$ oral load of glucose. Diabetes diagnoses were ascertained either from the longitudinal survey or from clinical records, as described previously [16]. Briefly, survey diagnoses were ascertained through research examinations using the World Health Organization criteria for epidemiological studies (i.e. a $2 \mathrm{~h}$ plasma glucose concentration $\geq 11.1 \mathrm{mmol} / 1$ [200 mg/dl]) [20]. Clinical diagnoses were ascertained through review of medical records of participants for any diabetes diagnosis between research examinations and were typically based on a $2 \mathrm{~h}$ glucose concentration $\geq 11.1 \mathrm{mmol} / 1$, a casual glucose concentration $\geq 11.1 \mathrm{mmol} / 1$ or a fasting glucose concentration meeting the prevailing criteria for diabetes diagnosis. The same methods were used to ascertain maternal and paternal diabetes prior to the birth of the participant (i.e. parental diabetes was diagnosed by OGTT or documented clinical diagnosis rather than by self-report). Data from maternal glucose tolerance tests during pregnancy were available for only $29 \%$ of participants; thus we did not analyse gestational diabetes defined on this basis. Moreover, we did not use fasting plasma glucose or $\mathrm{HbA}_{1 \mathrm{c}}$ to diagnose type 2 diabetes as these were not routinely measured in the early years of the study. Genotypic data were available for 211 established type 2 diabetes susceptibility genetic variants from a genome-wide association study, using methods described previously $[21,22]$.

Statistical analysis Participant and parental characteristics at the first research examination and the distribution of birthweight and BMI according to these characteristics were summarised using descriptive statistics (including median, 25 th and 75 th centiles for non-normally distributed variables), and compared using unpaired $t$ test, Wilcoxon rank-sum test, or $\chi^{2}$ test. Birthweight quintiles were generated using the PROC RANK statement in SAS. This procedure symmetrically divided an ordered distribution of the birthweight of all participants into five approximately equal strata. BMI was age- and sex-standardised to the study population to obtain a population-specific $z$ score. This standardisation procedure involved the following: (1) identifying sex-specific age-adjusted regression models that provide the best fit for BMI (i.e. $\log _{10} \mathrm{BMI}=\beta_{0}+\beta_{1}$ age $+\beta_{2}$ age $^{2}$ for male sex and $\log _{10}$ BMI $=\beta_{0}+\beta_{1}$ age $+\beta_{2}$ age $^{2}+\beta_{3}$ age $^{3}$ for female sex); (2) obtaining residuals of BMI at the first examination from sex-specific baseline models; (3) computing residuals of BMI at follow-up examinations from these baseline models and (4) standardising all residual values to obtain $z$ scores with mean $=0$ and $\mathrm{SD}=1$.

We constructed a type 2 diabetes susceptibility genetic risk score (GRS) for 211 established type 2 diabetes susceptibility genetic variants, which had been previously identified in type 2 diabetes genome-wide association studies of trans-ethnic populations [23], using methods described previously [21]. Briefly, the GRS was computed by summing the number of type 2 diabetes risk alleles, weighted by the logarithms of the published allelic odds ratios, across all 211 loci.

The period of risk began at the first research examination and ended with a diagnosis of diabetes or last biennial examination before the age of 40 years, whichever came first. A censoring age of 40 years was selected because there was little follow-up beyond 40 years of age among participants with known birthweights. Age- and sexspecific incidence rates of diabetes were computed per 1000 person-years of follow-up. To determine the effect of birthweight on diabetes incidence, Poisson regression models were constructed. Models were adjusted for sex, BMI $z$ score (fitted as time-varying), the GRS and maternal variables, including race/ethnicity, age at the birth of the participant and having diabetes prior to the birth of the participant. Multivariable models were not adjusted for paternal variables because of limited data.

Effects of birthweight on diabetes, assessed as incidence rate ratios (IRRs), were not uniform over time, and in some regression models the effects were $\mathrm{U}$ shaped. Therefore, all analyses were stratified by decade of age during follow-up, while in some analyses, birthweight was analysed as quintiles with the middle quintile as the reference category. When birthweight was analysed as a continuous variable, a quadratic term (the square of birthweight) was included in the model when statistically significant (at $p<0.05$ ) to account for the U shape. Models were tested for potential interactions of birthweight with significant covariates by including product terms. In a model comprising both linear and quadratic terms for birthweight, two product terms were fitted and a chunk test was conducted to determine their statistical significance. Models were also evaluated at different values of significant covariates to illustrate the joint effects of these variables with birthweight on the incidence of diabetes.

We investigated the interaction effects of two specific type 2 diabetes susceptibility genetic variants, maternally derived C allele of KCNQ1 SNP rs2299620 (the most significant type 2 diabetes susceptibility genetic variant in this study population) [24] and a novel R1420H in $A B C C 8$ (associated with both high birthweight and type 2 diabetes in this study population [14]), on the association between birthweight and type 2 diabetes.

Sensitivity analyses were conducted to ascertain the validity of risk estimates. This process included restricting data to participants who were not offspring of a diabetic woman to remove any confounding by exposure to an intrauterine diabetic environment. The effect of using BMI as the measure of adiposity rather than absolute weight and height was investigated by comparing estimates of these variables in regression models. A similar approach was used to 
Table 1 Participant characteristics and distributions of birthweight and BMI at the first research examination

\begin{tabular}{|c|c|c|c|c|c|}
\hline \multirow[t]{2}{*}{ Characteristic } & \multirow[t]{2}{*}{$n(\%)$} & \multicolumn{2}{|l|}{ Birthweight (kg) } & \multicolumn{2}{|l|}{ BMI $z$ score $^{\mathrm{a}}$} \\
\hline & & Median (Q1, Q3) & $p$ value & Mean (SD) & $p$ value \\
\hline Total & $3039(100)$ & $3.45(3.15,3.75)$ & - & $0.00(1.00)^{\mathrm{b}}$ & - \\
\hline \multicolumn{6}{|c|}{ Participant characteristics } \\
\hline \multicolumn{6}{|l|}{ Birthweight } \\
\hline Quintile 1 & $623(20.5)$ & $2.85(1.33,3.05)^{\mathrm{c}}$ & - & $-0.18(0.98)$ & \multirow[t]{5}{*}{$<0.001$} \\
\hline Quintile 2 & $590(19.4)$ & $3.20(3.06,3.34)^{\mathrm{c}}$ & - & $-0.17(1.04)$ & \\
\hline Quintile 3 & $630(20.7)$ & $3.45(3.34,3.55)^{\mathrm{c}}$ & - & $-0.03(0.97)$ & \\
\hline Quintile 4 & $576(19.0)$ & $3.69(3.55,3.85)^{\mathrm{c}}$ & - & $0.12(0.98)$ & \\
\hline Quintile 5 & $620(20.4)$ & $4.05(3.85,5.55)^{\mathrm{c}}$ & - & $0.27(0.96)$ & \\
\hline \multicolumn{6}{|l|}{ Sex } \\
\hline Male & $1336(44.0)$ & $3.52(3.18,3.84)$ & \multirow[t]{2}{*}{$<0.001$} & $0.00(1.00)^{\mathrm{b}}$ & \multirow[t]{2}{*}{-} \\
\hline Female & $1703(56.0)$ & $3.37(3.09,3.69)$ & & $0.00(1.00)^{\mathrm{b}}$ & \\
\hline \multicolumn{6}{|l|}{ Age, years } \\
\hline $5-9$ & $1960(64.5)$ & $3.45(3.15,3.77)$ & \multirow[t]{3}{*}{$<0.001$} & $-0.02(0.92)^{\mathrm{b}}$ & \multirow[t]{3}{*}{-} \\
\hline $10-14$ & $835(27.5)$ & $3.43(3.10,3.75)$ & & $0.09(1.13)^{\mathrm{b}}$ & \\
\hline$\geq 15$ & $244(8.0)$ & $3.35(3.01,3.65)$ & & $-0.15(1.08)^{\mathrm{b}}$ & \\
\hline \multicolumn{6}{|l|}{ Year of birth } \\
\hline 1960 and earlier & $395(13.0)$ & $3.38(3.10,3.67)$ & \multirow[t]{5}{*}{0.074} & $-0.40(0.85)$ & \multirow[t]{5}{*}{$<0.001$} \\
\hline $1961-1970$ & $781(25.7)$ & $3.45(3.15,3.75)$ & & $-0.13(0.90)$ & \\
\hline 1971-1980 & $666(21.9)$ & $3.45(3.15,3.75)$ & & $-0.04(1.04)$ & \\
\hline $1981-1990$ & $909(29.9)$ & $3.43(3.15,3.77)$ & & $0.21(1.02)$ & \\
\hline $1991-2000$ & $288(9.5)$ & $3.52(3.08,3.83)$ & & $0.33(1.03)$ & \\
\hline \multicolumn{6}{|c|}{ Type 2 diabetes GRS } \\
\hline Tertile 1 & $1013(33.3)$ & $3.43(3.12,3.75)$ & \multirow[t]{3}{*}{0.940} & $-0.05(0.99)$ & \multirow[t]{3}{*}{0.096} \\
\hline Tertile 2 & $1013(33.3)$ & $3.47(3.15,3.76)$ & & $0.01(0.98)$ & \\
\hline Tertile 3 & $1013(33.3)$ & $3.41(3.15,3.75)$ & & $0.04(1.02)$ & \\
\hline \multicolumn{6}{|l|}{ Maternal characteristics } \\
\hline \multicolumn{6}{|c|}{ Age at index birth, years } \\
\hline $0-18$ & $970(31.9)$ & $3.42(3.14,3.72)$ & \multirow[t]{3}{*}{0.003} & $0.04(0.95)$ & 0.489 \\
\hline $19-22$ & $1029(33.9)$ & $3.41(3.13,3.75)$ & & $-0.05(1.04)$ & \\
\hline$\geq 23$ & $1040(34.2)$ & $3.49(3.15,3.81)$ & & $0.01(1.00)$ & \\
\hline American Indian ar & & & & & \\
\hline Full & $2755(90.7)$ & $3.45(3.13,3.75)$ & 0.046 & $0.00(0.99)$ & 0.509 \\
\hline Not full & $284(9.3)$ & $3.49(3.19,3.81)$ & & $0.04(1.08)$ & \\
\hline Diabetes prior to in & & & & & \\
\hline Yes & $102(3.4)$ & $3.64(3.15,4.00)$ & 0.024 & $0.81(1.00)$ & $<0.001$ \\
\hline No & 2937 (96.6) & $3.45(3.15,3.75)$ & & $-0.03(0.99)$ & \\
\hline Paternal characteristic & & & & & \\
\hline Age at index birth, & & & & & \\
\hline$\leq 20$ & $764(28.5)$ & $3.41(3.13,3.73)$ & 0.010 & $0.01(1.00)$ & 0.655 \\
\hline $21-30$ & $1519(56.6)$ & $3.45(3.15,3.77)$ & & $0.04(1.00)$ & \\
\hline$\geq 31$ & $401(14.9)$ & $3.46(3.13,3.77)$ & & $-0.04(0.97)$ & \\
\hline American Indian ar & & & & & \\
\hline Full & $2238(82.0)$ & $3.45(3.15,3.75)$ & 0.165 & $0.01(1.00)$ & 0.134 \\
\hline Not full & $490(18.0)$ & $3.40(3.12,3.73)$ & & $0.08(1.01)$ & \\
\hline Diabetes prior to in & & & & & \\
\hline Yes & $88(4.1)$ & $3.34(3.05,3.70)$ & 0.063 & $0.22(1.23)$ & 0.040 \\
\hline No & $2064(95.9)$ & $3.45(3.15,3.75)$ & & $0.00(0.97)$ & \\
\hline
\end{tabular}

${ }^{a}$ Logarithm of BMI was age- and sex-standardised to the study population to obtain $z$ score

${ }^{\mathrm{b}}$ Mean of 0 and SD of 1 are due to age and sex standardisation

${ }^{\mathrm{c}}$ Data are summarised as median and range

${ }^{\mathrm{d}}$ Data on age at index birth and race/ethnicity were available for 2684 individuals $(88.3 \%)$ and on diabetes prior to index birth for 2728 individuals $(89.8 \%)$

Q1, quartile 1; Q3, quartile 3

compare the effect of using weighted GRS rather than raw (all alleles weighted equally) GRS. Moreover, we compared risk estimates obtained from incidence analyses where the period of risk began at the first research examination when the participant did not have diabetes, with those obtained from incidence analyses where the period of risk began at birth and included participants who had diabetes at their first examinations. 
All analyses were performed using SAS (version 9.4; SAS Institute, Cary, NC, USA). A two-sided $p<0.05$ was considered statistically significant.

\section{Results}

\section{Participant characteristics at the first research examination} This study comprised 3039 participants (56\% female sex, median birthweight $3.45 \mathrm{~kg}$, $3 \%$ offspring of women with type 2 diabetes; Table 1). Male participants, participants who were younger or participants who were offspring of older fathers, of a diabetic mother or of mothers who were older or not full American Indians, were heavier at birth $(p<0.05$; Table 1$)$. At the first research examination (median age 8.6 years, $\mathrm{Q} 1=6.8$, $\mathrm{Q} 3=11.4$ ), participants with greater birthweight, offspring of a diabetic mother, and offspring of fathers with diabetes before the birth of the child, had greater BMI $z$ scores $(p<0.05$; Table 1). Similarly, participants who were born more recently had greater BMI $z$ scores $(p<0.001$; see ESM Table 1 for secular trends in other participant characteristics). Tertiles of the GRS for 211 established type 2 diabetes susceptibility genetic variants were not significantly related to weight at birth or BMI $z$ score at the first examination.

Diabetes incidence Among 3039 participants who were followed for a median of 14.3 years (quartile $1=8.0$, quartile $3=24.3$ ) from the first research examination, there were 652 incident cases of diabetes (13.4 per 1000 person-years). Of these incident cases, 420 (64.4\%) were ascertained through the longitudinal survey and 232 (35.6\%) through review of clinical records. Crude diabetes cumulative incidence (Fig. 1) and incidence density (Table 2) increased with age and were greater in participants in the lowest and the highest birthweight quintiles. Comparing diabetes incidence in these two high-risk

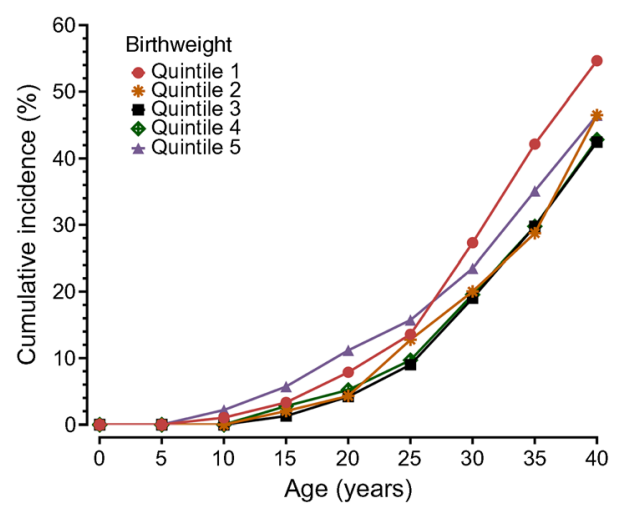

Fig. 1 Crude cumulative incidence (incidence proportion from the first research examination) of type 2 diabetes by birthweight quintile. Cumulative incidence (unadjusted for covariates), where the period of risk began at the first research examination when the participant did not have diabetes, was computed independently in each group of participants defined by birthweight quintile groups, those in the highest birthweight quintile had a greater cumulative incidence from age 10 years to 25 years, after which their cumulative incidence was surpassed by those in the lowest birthweight quintile (Fig. 1). This time-varying effect of birthweight on diabetes risk persisted when adjusted for sex, year of birth, GRS, maternal race/ethnicity and maternal age at the birth of the participant, with a U-shaped effect of birthweight on diabetes incidence at $10-19$ years $(p<0.001)$ but inverse linear effects at 20-29 years $(p=0.027)$ and at 30-39 years $(p=0.042)$ (Fig. 2a-c). After further adjustment for BMI and maternal diabetes, the effect of birthweight on diabetes incidence remained $U$ shaped, with greater risk observed in participants with the lowest (IRR 1.79 [95\% CI $1.02,3.14]$ ) and highest (IRR 1.50 [95\% CI 0.87, 2.59]) birthweight quintiles (Fig. 2d). After this adjustment, the inverse linear effects at older ages were more pronounced ( $p<0.001$ at $20-29$ years and $p=0.003$ at 30-39 years), with a greater risk observed in participants in lower birthweight quintiles only (20-29 years, quintile 1: IRR 1.50 [95\% CI 1.05 , 2.15]; 30-39 years, quintile 1: IRR 1.50 [95\% CI 1.04, 2.16] and quintile 2: IRR 1.16 [95\% CI 0.79, 1.71]) (Fig. 2e, f). The effect of birthweight on diabetes incidence could not be reliably determined at age 5-9 years because there were only seven incident cases of diabetes (Table 2).

Other factors that increased diabetes risk included greater BMI $z$ score, higher GRS for type 2 diabetes and maternal diabetes (ESM Table 2). These covariates did not confound or interact with the effect of birthweight on diabetes incidence at all age intervals ( $p \geq 0.05$ for all interactions), although they independently added information about diabetes incidence. This was illustrated by results of a model of diabetes IRRs evaluated at age 1019 years for variables such as increasing quintiles of birthweight, increasing tertiles of BMI $z$ score, a GRS below or above the median and the presence or absence of maternal diabetes in pregnancy (Fig. 3). While the rate ratios were uniform, the absolute rate differences were, of course, much greater in those at greater risk from these variables, especially maternal diabetes.

However, there was no difference in the incidence ratios associated with birthweight categories between those with and without exposure to maternal diabetes, as illustrated in Fig. 3 (ratios comparing incidence rates shown in Fig. $3 \mathrm{a}$ vs Fig. $3 \mathrm{~b}$ are the same as those comparing rates in Fig. 3c vs Fig. 3d). Type 2 diabetes GRS predicted diabetes incidence in each age group, with or without adjustment for the other diabetes risk factors (IRRs ranged from 1.30 to 1.40 per SD of the GRS; ESM Table 2).

Since the GRS was based on genetic variants associated with type 2 diabetes in non-American Indian populations, variations in $A B C C 8$ and $K C N Q 1$, which have a large effect on type 2 diabetes in the present study population, were also analysed. Estimates of the effect of birthweight on diabetes 
Table 2 Crude incidence rates of diabetes by birthweight quintiles and age group during follow-up

\begin{tabular}{|c|c|c|c|c|c|c|c|c|c|c|c|c|c|c|c|}
\hline \multirow[t]{2}{*}{ Age (years) } & \multicolumn{3}{|c|}{ Quintile 1} & \multicolumn{3}{|c|}{ Quintile 2} & \multicolumn{3}{|l|}{ Quintile 3} & \multicolumn{3}{|c|}{ Quintile 4} & \multicolumn{3}{|c|}{ Quintile 5} \\
\hline & PY & $n$ & Rate $^{\mathrm{a}}$ & PY & $n$ & Rate $^{\mathrm{a}}$ & PY & $n$ & Rate $^{\mathrm{a}}$ & PY & $n$ & Rate $^{a}$ & PY & $n$ & Rate $^{a}$ \\
\hline $0-9$ & 953.5 & 2 & 2.1 & 976.5 & 0 & 0.0 & 1024.3 & 0 & 0.0 & 1049.9 & 0 & 0.0 & 1123.6 & 5 & 4.5 \\
\hline $10-19$ & 4321.7 & 31 & 7.2 & 4318.7 & 19 & 4.4 & 4657.7 & 20 & 4.3 & 4104.6 & 22 & 5.4 & 4414.8 & 42 & 9.5 \\
\hline $20-29$ & 3126.0 & 69 & 22.1 & 2827.2 & 51 & 18.0 & 3445.6 & 55 & 16.0 & 2671.2 & 41 & 15.4 & 2653.4 & 38 & 14.3 \\
\hline $30-39$ & 1432.5 & 67 & 46.8 & 1450.1 & 52 & 35.9 & 1593.9 & 52 & 32.6 & 1318.6 & 43 & 32.6 & 1230.0 & 43 & 35.0 \\
\hline Total & 9833.7 & 169 & 19.9 & 9572.4 & 122 & 12.7 & $10,721.5$ & 127 & 11.8 & 9144.3 & 106 & 11.6 & 9421.9 & 128 & 13.5 \\
\hline
\end{tabular}

${ }^{a}$ Incidence rate of diabetes per 1000 person-years obtained by counting person-years accumulated in each decade of age

PY, person-years of follow-up

incidence in models adjusted for the single variants, a novel $\mathrm{R} 1420 \mathrm{H}$ in $A B C C 8$ and rs2299620 in KCNQ1 (ESM Table 3), were similar to those of models adjusted for type 2 diabetes

GRS (ESM Table 2). However, for $A B C C 8$, there was a
Fig. 2 Effect of birthweight on the risk of type 2 diabetes. $(\mathbf{a}-\mathbf{c})$ Risk of type 2 diabetes (adjusted for all participant and maternal characteristics except BMI of participant and maternal diabetes) at age $10-19$ years $(\mathbf{a} ; n=2912$, $p<0.001), 20-29$ years (b; $n=$ $1989, p=0.027$ ) and 30-39 years $(\mathbf{c} ; n=1079, p=0.042) .(\mathbf{d}-\mathbf{f})$

Risk of type 2 diabetes (adjusted for all participant and maternal characteristics including BMI of participant and maternal diabetes) at age $10-19$ years $(\mathbf{d} ; n=2912$, $p<0.001), 20-29$ years $(\mathbf{e} ; n=$ $1989, p<0.001)$ and $30-39$ years (f; $n=1079, p=0.003$ ). The height of each bar represents the point estimate of the IRR and the error bars represent its $95 \% \mathrm{CI}$
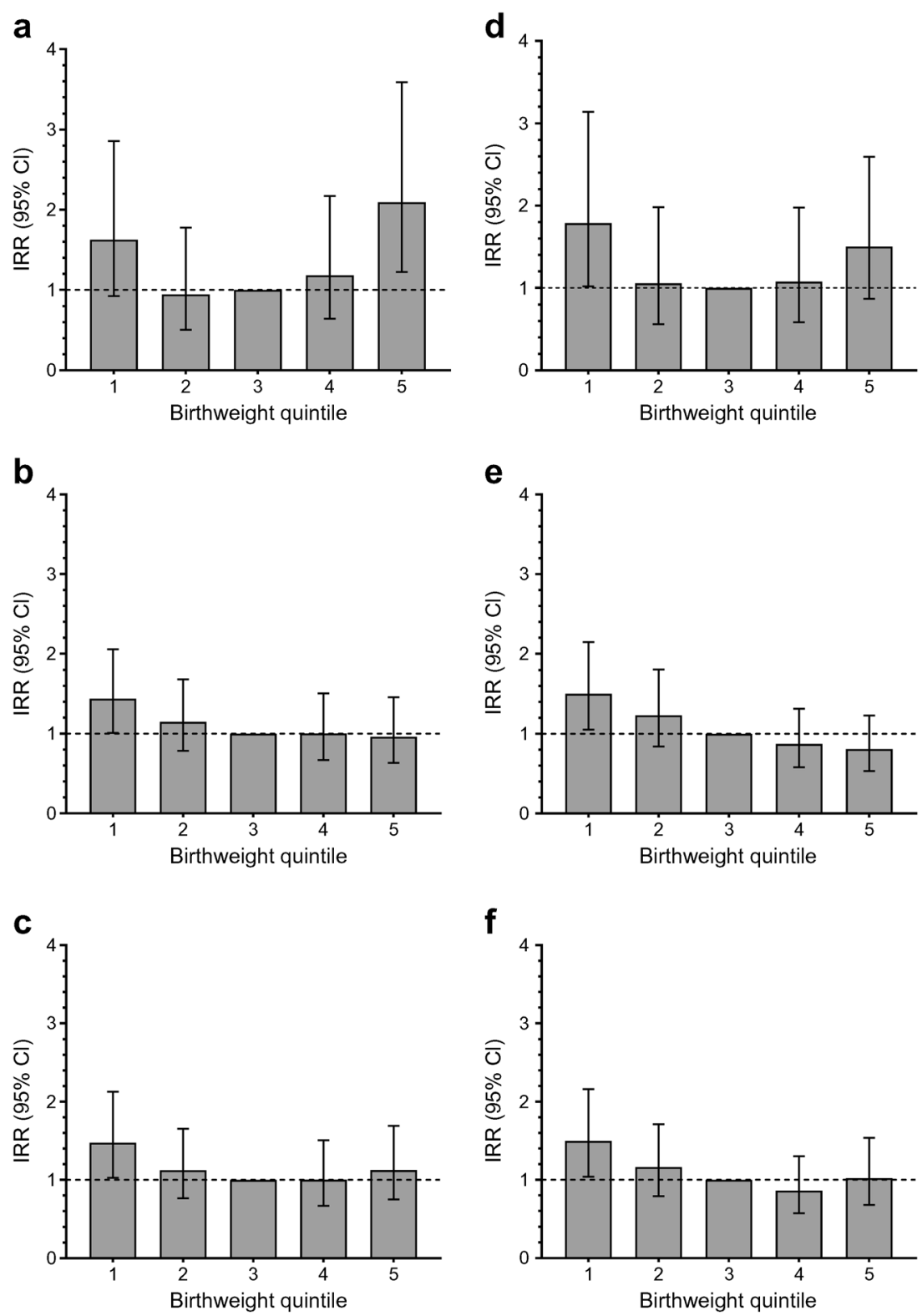
Fig. 3 Joint effects of BMI, type 2 diabetes GRS and maternal diabetes with birthweight on the incidence rate (per 1000 personyears) of type 2 diabetes at age $10-19$ years. (a) Offspring of a non-diabetic woman with a low (below the median) type 2 diabetes GRS. (b) Offspring of a non-diabetic woman with a high (at or above the median) type 2 diabetes GRS. (c) Offspring of a diabetic woman with a low type 2 diabetes GRS. (d) Offspring of a diabetic woman with a high type 2 diabetes GRS. py, person-years
BMI $z$ score -2
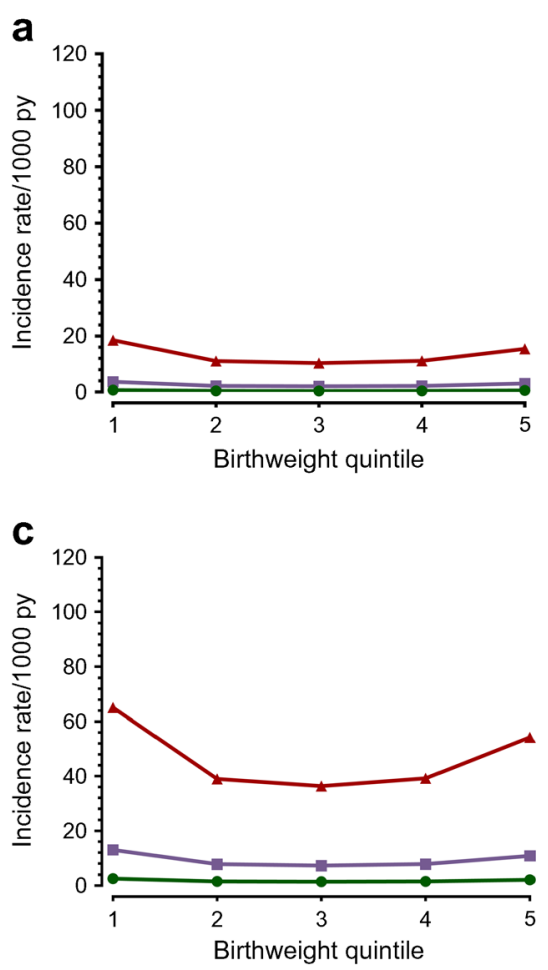

BMI z score 0

b

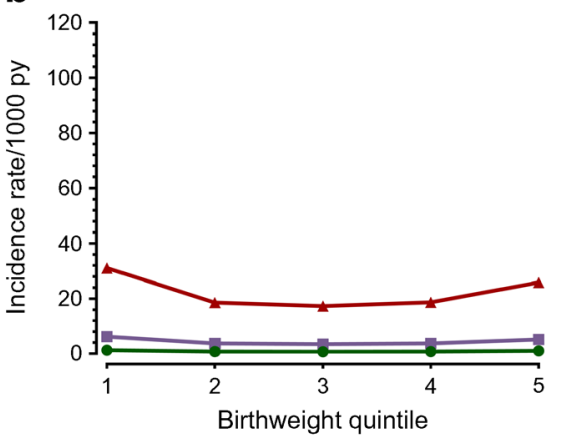

d

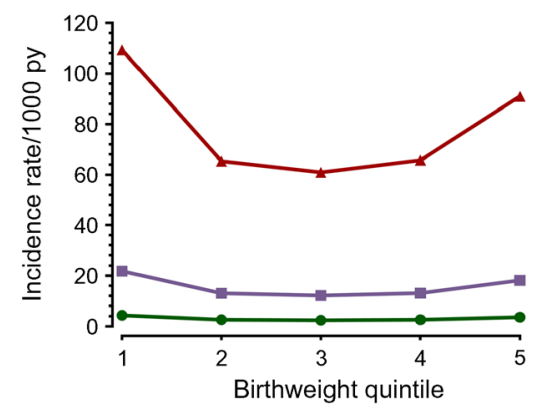

significant interaction effect of the R1420H variant with the association between birthweight and diabetes incidence at age 20-29 years $(p=0.006)$, with a positive linear effect of birthweight on diabetes incidence at 20-29 years among heterozygous carriers of the $\mathrm{H}$ allele (IRR 2.68 per $\mathrm{kg}$ [95\% CI $1.09,6.60] ; n=108$ ) and a negative linear effect among noncarriers of the $\mathrm{H}$ allele (IRR 0.60 per $\mathrm{kg}$ [95\% CI 0.46, 0.77]; $n=2931)$. However, there was no significant interaction of the $A B C C 8 \mathrm{R} 1420 \mathrm{H}$ variant with the effect of birthweight on type 2 diabetes incidence at age 10-19 years or 30-39 years. The $K C N Q 1$ variant did not interact with the effect of birthweight on type 2 diabetes incidence in any age group.

Sensitivity analyses Estimates of models restricted to data from participants who were offspring of a non-diabetic woman were similar to those including all participants. Comparing effects of two measures of adiposity, there were no meaningful differences between models comprising BMI and those comprising absolute weight and height. Similarly, findings of regression models on the association of weighted GRS with birthweight and type 2 diabetes incidence, and on the impact of weighted GRS on birthweight-associated type 2 diabetes risk, were similar to those of models in which raw GRS was used. Moreover, in analyses in which the period of risk began at birth (ESM Fig. 1), estimates of the effect of birthweight on diabetes incidence were similar to those observed in analyses where the period of risk began at the first research examination without diabetes (Fig. 1).

\section{Discussion}

Statement of principal findings Birthweight had differential effects on early-onset diabetes, with the lowest and highest birthweight quintiles increasing type 2 diabetes risk during adolescence (10-19 years). In contrast to the effect of low birthweight, the effect of high birthweight on annual incidence rates of type 2 diabetes did not persist into adulthood (2039 years), although the cumulative incidence remained greater in this quintile than in the middle three quintiles because of the greater incidence of type 2 diabetes in the earlier years. An aggregate of 221 established type 2 diabetes susceptibility genetic variants that were identified in genome-wide studies of non-American Indian populations independently predicted the risk of early-onset type 2 diabetes and added to the effect of birthweight on type 2 diabetes risk in our south-western American Indian population. However, it was not significantly associated with birthweight and did not confound or modify the effect of birthweight on type 2 diabetes risk. Similarly, greater BMI during follow-up and being exposed to a diabetic intrauterine environment independently predicted type 2 diabetes risk and added to the effect of birthweight on type 2 diabetes risk.

Strengths and weaknesses of the study The strength of this study is its prospective and long-term ascertainment of diabetes using definitive tests of blood glucose control. This allows for a more reliable estimate of the incidence and the time of 
diabetes onset, thereby helping to clarify inconsistencies in existing evidence. However, our study is also limited by the non-adjustment of birthweight for the length at birth and gestational age, due to the lack of reliable data on these two covariates. As data from maternal glucose tolerance tests during the third trimester were not generally available, the maternal diabetes status included in our analyses largely reflects diabetes occurring prior to pregnancy, rather than gestational diabetes. However, in this study population, offspring of mothers with overt diabetes (largely developing before pregnancy) have a much greater incidence of early-onset type 2 diabetes than offspring whose mothers had impaired glucose tolerance $(7.8-11.0 \mathrm{mmol} / \mathrm{l})$ in the third trimester that is typical of gestational diabetes [25]. We did not report data on other indicators of abormal glucose regulation, such as plasma insulin levels, which were not measured throughout the study. However, in a previous analysis of children and young adults (aged 5-29 years) from this study population, birthweight was negatively associated with both fasting and $2 \mathrm{~h}$ insulin levels [26].

\section{Strength and weaknesses in relation to other studies Previous} studies of the association between birthweight and type 2 diabetes were largely conducted in older adults ( $\geq 40$ years) and findings were based on prevalence data which do not account for the time of diabetes onset. In most of these prior studies, low birthweight (but not high birthweight) was associated with greater type 2 diabetes risk [3, 4]. However, in a few studies, high (but not low) birthweight [27], or both low and high birthweight $[2,28,29]$, were associated with greater type 2 diabetes risk. In a study of adults in Mysore, India, the prevalence of diabetes was not significantly related to birthweight but was greatest in those who were short at birth and had greater ponderal index (birthweight/length ${ }^{3}$ ) [30]. The U-shaped effect of birthweight on adolescence-onset type 2 diabetes risk observed in the present study was also seen in our previous study of the prevalence of type 2 diabetes in Pima Indians [2]; the present study extends these observations in showing that the increased risk associated with high birthweight occurs primarily at younger ages. A U-shaped association was also observed in prior studies conducted in children (aged $\leq 18$ years) from Taiwan and a Canadian indigenous population $[28,29]$. In China, where there is a relatively high prevalence and increasing incidence of early-onset type 2 diabetes [31], birthweight had a U-shaped association with fasting plasma insulin and HOMA-IR levels among adolescents (mean age 15 years) [32]. Similarly, the association between high birthweight and greater type 2 diabetes risk observed in the present study was also observed in a study conducted among children and adults (aged 10-44 years) in a Canadian indigenous population with a high prevalence of type 2 diabetes [29]. Findings of the present and those of prior studies suggest that high birthweight (in addition to low birthweight) has an impact on type 2 diabetes risk during childhood, especially in populations with an exceptionally high type 2 diabetes prevalence.

Meaning of the study: possible explanations and implications for clinicians and policy makers The association between low birthweight and greater type 2 diabetes risk has been attributed to several factors: (1) the effects of nutritional insufficiency in utero on fetal growth and fetal programming of the endocrine system [7, 33]; (2) selective survival of small babies prone to insulin resistance [2] and (3) genetic variants mediating fetal growth and impairment in insulin secretion or sensitivity [9, 34, 35]. The observed U-shaped association between birthweight and the risk of adolescence-onset diabetes may be driven by the high prevalence of maternal diabetesmediated fetal macrosomia in this study population [36]. This association was also observed in a Canadian aboriginal population with a high prevalence of type 2 diabetes in children and young adults $[27,29]$. These findings highlight the role of gene-intrauterine environment interaction on the association between high birthweight and later type 2 diabetes risk.

The additional effect of higher GRS, greater BMI during follow-up and exposure to a diabetic intrauterine environment on the incidence of early-onset diabetes in the present study conforms with our previous discovery of a trans-generational cycle of obesity and type 2 diabetes [37]. In this vicious cycle, offspring of a diabetic woman have a greater risk of early obesity and type 2 diabetes, especially during their reproductive age. When this occurs in female offspring of a diabetic woman, the cycle repeats, thereby causing an increasing prevalence of type 2 diabetes in subsequent generations. This finding emphasises the need for preventing or delaying diabetes in women before or during their childbearing years. Given the increasing incidence of early-onset type 2 diabetes and its preponderance in the female sex in most regions of the world and across all race/ethnicities [6], the current findings are highly relevant to other populations. Specifically, our findings may have direct applications to populations with a high prevalence of type 2 diabetes among youth and young adults, such as the indigenous populations of the USA, Canada, Australia and New Zealand [5, 38, 39], and the Chinese population [31].

Unanswered questions and future research Genetic variants underlying the association between birthweight and type 2 diabetes are still not well understood. In the present study, aggregate contribution of common type 2 diabetes susceptibility genetic variants identified in trans-ethnic populations, including variants also known to be associated with birthweight (e.g. ADCY5, CDKAL1, HHEX-IDE, ANK1, $M T N R 1 B$ and $A B C C 8$ ) [12-14], did not modify the effect of birthweight on type 2 diabetes risk. However, a novel R1420H variant in $A B C C 8$, reported to have a large effect on the risk of type 2 diabetes and high birthweight in Pima Indians [14], 
significantly modified the direction of effect of birthweight on type 2 diabetes incidence at age 20-29 years, with a negative linear effect observed in participants without the $A B C C 8$ risk genotype and a positive linear effect in those with the $A B C C 8$ genotype. However, this finding did not replicate in other age groups and may be limited by the small number of diabetes cases observed $(n=42)$ in the 108 participants with the $A B C C 8$ mutation. Therefore, further investigation into the genetic mechanisms underlying these associations is needed. For example, this may involve exploring the role of altered gene expression mediated by epigenetic modifications in an adverse intrauterine environment [40].

\section{Conclusion}

We report a novel finding of differential effects of birthweight on type 2 diabetes risk in a population with a high prevalence of early-onset (aged $<40$ years) type 2 diabetes, in which birthweight had a U-shaped effect on type 2 diabetes risk in adolescents (aged 10-19 years) but a negative linear effect in young adults (aged 20-39 years). Higher type 2 diabetes GRS, greater BMI and being an offspring of a diabetic woman added to the risk of early-onset type 2 diabetes but did not modify or explain the effect of birthweight on type 2 diabetes incidence. High birthweight was associated with markedly elevated risk of type 2 diabetes at age 10-19 years, but not at older ages. This suggests that the effect of high birthweight on type 2 diabetes risk, reported only in populations with a high prevalence of type 2 diabetes, occurs largely at young ages and may be driven by the inter-generational vicious cycle of type 2 diabetes and obesity in these populations [37].

Acknowledgements This research was supported by the Intramural Research Program of the National Institute of Diabetes and Digestive and Kidney Diseases (NIDDK). We are grateful to the NIDDK Phoenix clinic staff and to the residents of the Gila River Indian Community in Arizona for their participation in the study.

Data availability Data are available on request from the authors.

Funding This work was supported by the Intramural Research Program of the National Institute of Diabetes and Digestive and Kidney Diseases.

Duality of interest The authors declare that there is no duality of interest associated with this manuscript.

Contribution statement MTO contributed to the conception and design of the study, analysed and interpreted data and prepared the first draft of the manuscript. LEW and SK contributed to the analysis and interpretation of data. RLH contributed to the acquisition, analysis and interpretation of data. MS contributed to the conception and design of the study and interpretation of data. RGN and LJB contributed to the acquisition and interpretation of data. WCK contributed to the conception and design of the study and to acquisition, analysis and interpretation of data. All authors revised the manuscript for intellectual content and approved the manuscript for submission. WCK is the guarantor of this work.

\section{References}

1. Hales CN, Barker DJ, Clark PM et al (1991) Fetal and infant growth and impaired glucose tolerance at age 64. BMJ 303(6809):1019 1022. https://doi.org/10.1136/bmj.303.6809.1019

2. McCance DR, Pettitt DJ, Hanson RL, Jacobsson LT, Knowler WC, Bennett PH (1994) Birth weight and non-insulin dependent diabetes: thrifty genotype, thrifty phenotype, or surviving small baby genotype? BMJ 308(6934):942-945. https://doi.org/10.1136/bmj. 308.6934 .942

3. Harder T, Rodekamp E, Schellong K, Dudenhausen JW, Plagemann A (2007) Birth weight and subsequent risk of type 2 diabetes: a meta-analysis. Am J Epidemiol 165(8):849-857. https:// doi.org/10.1093/aje/kwk071

4. Whincup PH, Kaye SJ, Owen CG et al (2008) Birth weight and risk of type 2 diabetes: a systematic review. JAMA 300(24):2886-2897. https://doi.org/10.1001/jama.2008.886

5. Mayer-Davis EJ, Dabelea D, Lawrence JM (2017) Incidence trends of type 1 and type 2 diabetes among youths, 2002-2012. N Engl J Med 377(3):301-1429. https://doi.org/10.1056/NEJMoa1610187

6. Lascar N, Brown J, Pattison H, Barnett AH, Bailey CJ, Bellary S (2018) Type 2 diabetes in adolescents and young adults. Lancet Diabetes Endocrinol 6(1):69-80. https://doi.org/10.1016/S22138587(17)30186-9

7. Hales CN, Barker DJ (1992) Type 2 (non-insulin-dependent) diabetes mellitus: the thrifty phenotype hypothesis. Diabetologia 35(7):595-601. https://doi.org/10.1007/BF00400248

8. Neel JV (1962) Diabetes mellitus: a "thrifty" genotype rendered detrimental by "progress"? Am J Hum Genet 14(4):353-362

9. Hattersley AT, Tooke JE (1999) The fetal insulin hypothesis: an alternative explanation of the association of low birthweight with diabetes and vascular disease. Lancet 353(9166):1789-1792. https://doi.org/10.1016/S0140-6736(98)07546-1

10. Lindsay RS, Dabelea D, Roumain J, Hanson RL, Bennett PH, Knowler WC (2000) Type 2 diabetes and low birth weight: the role of paternal inheritance in the association of low birth weight and diabetes. Diabetes 49(3):445-449. https://doi.org/10.2337/diabetes. 49.3.445

11. Hypponen E, Smith GD, Power C (2003) Parental diabetes and birth weight of offspring: intergenerational cohort study. BMJ 326(7379):19-20. https://doi.org/10.1136/bmj.326.7379.19

12. Horikoshi M, Beaumont RN, Day FR et al (2016) Genome-wide associations for birth weight and correlations with adult disease. Nature 538(7624):248-252. https://doi.org/10.1038/nature19806

13. Zeng CP, Chen YC, Lin X et al (2017) Increased identification of novel variants in type 2 diabetes, birth weight and their pleiotropic loci. J Diabetes 9(10):898-907. https://doi.org/10.1111/1753-0407. 12510

14. Baier LJ, Muller YL, Remedi MS et al (2015) ABCC8 R1420H loss-of-function variant in a Southwest American Indian community: association with increased birth weight and doubled risk of type 2 diabetes. Diabetes 64(12):4322-4332. https://doi.org/10.2337/ db15-0459

15. Bennett PH, Burch TA, Miller M (1971) Diabetes mellitus in American (Pima) Indians. Lancet 2(7716):125-128

16. Knowler WC, Bennett PH, Hamman RF, Miller M (1978) Diabetes incidence and prevalence in Pima Indians: a 19-fold greater incidence than in Rochester, Minnesota. Am J Epidemiol 108(6):497505. https://doi.org/10.1093/oxfordjournals.aje.a112648

17. Pavkov ME, Hanson RL, Knowler WC, Bennett PH, Krakoff J, Nelson RG (2007) Changing patterns of type 2 diabetes incidence among Pima Indians. Diabetes Care 30(7):1758-1763. https://doi. org $/ 10.2337 / \mathrm{dc} 06-2010$

18. Dabelea D, Hanson RL, Bennett PH, Roumain J, Knowler WC, Pettitt DJ (1998) Increasing prevalence of type II diabetes in 
American Indian children. Diabetologia 41(8):904-910. https://doi. org/10.1007/s001250051006

19. Dabelea D, Palmer JP, Bennett PH, Pettitt DJ, Knowler WC (1999) Absence of glutamic acid decarboxylase antibodies in Pima Indian children with diabetes mellitus. Diabetologia 42(10):1265-1266

20. WHO Study Group on Diabetes Mellitus and World Health Organization (1985) Diabetes mellitus: report of a WHO study group [meeting held in Geneva from 11 to 16 February 1985]. WHO, Geneva Available from www.who.int/iris/handle/10665/ 39592

21. Hanson RL, Rong R, Kobes S et al (2015) Role of established type 2 diabetes-susceptibility genetic variants in a high prevalence American Indian population. Diabetes 64(7):2646-2657. https:// doi.org/10.2337/db14-1715

22. Piaggi P, Masindova I, Muller YL et al (2017) A genome-wide association study using a custom genotyping array identifies variants in GPR158 associated with reduced energy expenditure in American Indians. Diabetes 66(8):2284-2295. https://doi.org/10. 2337/db16-1565

23. Mahajan A, Talium D, Thurner M et al (2018) Fine-mapping type 2 diabetes loci to single-variant resolution using high-density imputation and islet-specific epigenome maps. Nat Genet 50(11):15051513. https://doi.org/10.1038/s41588-018-0241-6

24. Hanson RL, Guo T, Muller YL et al (2013) Strong parent-of-origin effects in the association of KCNQ1 variants with type 2 diabetes in American Indians. Diabetes 62(8):2984-2991. https://doi.org/10. 2337/db12-1767

25. Franks PW, Looker HC, Kobes S et al (2006) Gestational glucose tolerance and risk of type 2 diabetes in young Pima Indian offspring. Diabetes 55(2):460-465. https://doi.org/10.2337/diabetes. 55.02.06.db05-0823

26. Dabelea D, Pettitt DJ, Hanson RL, Imperatore G, Bennett PH, Knowler WC (1999) Birth weight, type 2 diabetes, and insulin resistance in Pima Indian children and young adults. Diabetes Care 22(6):944-950. https://doi.org/10.2337/diacare.22.6.944

27. Dyck RF, Klomp H, Tan L (2001) From "thrifty genotype" to "hefty fetal phenotype": the relationship between high birthweight and diabetes in Saskatchewan registered Indians. Can J Public Health 92(5):340-344

28. Wei J-N, Sung F-C, Li C-Y et al (2003) Low birth weight and high birth weight infants are both at an increased risk to have type 2 diabetes among schoolchildren in Taiwan. Diabetes Care 26(2): 343-348. https://doi.org/10.2337/diacare.26.2.343

29. Young TK, Martens PJ, Taback SP et al (2002) Type 2 diabetes mellitus in children: prenatal and early infancy risk factors among native Canadians. Arch Pediatr Adolesc Med 156(7):651-655. https://doi.org/10.1001/archpedi.156.7.651

30. Fall CH, Stein CE, Kumaran K et al (1998) Size at birth, maternal weight, and type 2 diabetes in South India. Diabet Med 15(3):220 227. https://doi.org/10.1002/(SICI)1096-9136(199803)15

31. Wang L, Gao P, Zhang $\mathrm{M}$ et al (2017) Prevalence and ethnic pattern of diabetes and prediabetes in China in 2013. JAMA 317(24): 2515-2523. https://doi.org/10.1001/jama.2017.7596

32. Tam CHT, Wang Y, Luan J et al (2015) Non-linear relationship between birth weight and cardiometabolic risk factors in Chinese adolescents and adults. Diabet Med 32(2):220-225. https://doi.org/ 10.1111/dme. 12630

33. Barker DJ (1990) The fetal and infant origins of adult disease. BMJ 301(6761):1111. https://doi.org/10.1136/bmj.301.6761.1111

34. Frayling TM, Hattersley AT (2001) The role of genetic susceptibility in the association of low birth weight with type 2 diabetes. $\mathrm{Br}$ Med Bull 60(1):89-101. https://doi.org/10.1093/bmb/60.1.89

35. Hughes AE, Nodzenski M, Beaumont RN et al (2018) Fetal genotype and maternal glucose have independent and additive effects on birth weight. Diabetes 67(5):1024-1029. https://doi.org/10.2337/ db17-1188

36. Pettitt DJ, Knowler WC, Baird HR, Bennett PH (1980) Gestational diabetes: infant and maternal complications of pregnancy in relation to third-trimester glucose tolerance in the Pima Indians. Diabetes Care 3(3):458-464. https://doi.org/10.2337/diacare.3.3.458

37. Pettitt DJ, Knowler WC (1988) Diabetes and obesity in the Pima Indians: a cross-generational vicious cycle. J Obesity Weight Regul 7:61-65

38. Australian Bureau of Statistics (2013). Australian aboriginal and Torres Strait Island health survey: first results, Australia, 2012 2013. Available from http://www.abs.gov.au/ausstats/abs@.nsf/ $\mathrm{mf} / 4727.0 .55 .001$, accessed 10 October 2018

39. Chamberlain C, McNamara B, Williams ED et al (2013) Diabetes in pregnancy among indigenous women in Australia, Canada, New Zealand and the United States: a systematic review of the evidence for screening in early pregnancy. Diabetes Metab Res 29(4):241256. https://doi.org/10.1002/dmrr.2389

40. Chen P, Piaggi P, Traurig M et al (2017) Differential methylation of genes in individuals exposed to maternal diabetes in utero. Diabetologia 60(4):645-655. https://doi.org/10.1007/s00125-0164203-1

Publisher's note Springer Nature remains neutral with regard to jurisdictional claims in published maps and institutional affiliations. 